\title{
Metastasis and Mortality in Men With Low- and Intermediate-Risk Prostate Cancer on Active Surveillance
}

\author{
P. Travis Courtney, MD, MAS 1,2; Rishi Deka, PhD 1,2; Nikhil V. Kotha, BS ${ }^{1,2}$; Daniel R. Cherry, MD, MAS 1,2 ; \\ Mia A. Salans, MD, MAS 1,2; Tyler J. Nelson, BS ${ }^{1,2}$; Abhishek Kumar, MD, MAS 1,2; Elaine Luterstein, BS ${ }^{1,2}$; \\ Anthony T. Yip, MD, MAS ${ }^{2}$; Vinit Nalawade, MS'2 J. Kellogg Parsons, MD, MHS 1,3,4; A. Karim Kader, MD, PhD ${ }^{1,3}$; \\ Tyler F. Stewart, MD ${ }^{1,4}$; and Brent S. Rose, MD ${ }^{1,2}$
}

\section{ABSTRACT}

Background: Active surveillance (AS) is a safe treatment option for men with low-risk, localized prostate cancer. However, the safety of AS for patients with intermediate-risk prostate cancer remains unclear. Patients and Methods: We identified men with NCCNclassified low-risk and favorable and unfavorable intermediate-risk prostate cancer diagnosed between 2001 and 2015 and initially managed with $\mathrm{AS}$ in the Veterans Health Administration. We analyzed progression to definitive treatment, metastasis, prostate cancerspecific mortality (PCSM), and all-cause mortality using cumulative incidences and multivariable competing-risks regression. Results: The cohort included 9,733 men, of whom 1,007 (10.3\%) had intermediate-risk disease (773 [76.8\%] favorable, 234 [23.2\%] unfavorable), followed for a median of 7.6 years. The 10 -year cumulative incidence of metastasis was significantly higher for patients with favorable $(9.6 \% ; 95 \% \mathrm{Cl}, 7.1 \%-12.5 \% ; P<.001)$ and unfavorable intermediaterisk disease $(19.2 \% ; 95 \% \mathrm{Cl}, 13.4 \%-25.9 \% ; P<.001)$ than for those with low-risk disease (1.5\%; $95 \% \mathrm{Cl}, 1.2 \%-1.9 \%)$. The 10-year cumulative incidence of PCSM was also significantly higher for patients with favorable (3.7\%; $95 \% \mathrm{Cl}, 2.3 \%-5.7 \% ; P<.001)$ and unfavorable intermediate-risk disease $(11.8 \% ; 95 \% \mathrm{Cl}, 6.8 \%-18.4 \% ; P<.001)$ than for those with low-risk disease (1.1\%; $95 \% \mathrm{Cl}, 0.8 \%-1.4 \%)$. In multivariable competing-risks regression, favorable and unfavorable intermediate-risk patients had significantly increased risks of metastasis and PCSM compared with low-risk patients (all $P<.001$ ). Conclusions: Compared with low-risk patients, those with favorable and unfavorable intermediate-risk prostate cancer managed with AS are at increased risk of metastasis and PCSM. AS may be an appropriate option for carefully selected patients with favorable intermediate-risk prostate cancer, though identification of appropriate candidates and AS protocols should be tested in future prospective studies.

J Natl Compr Canc Netw 2022;20(2):151-159 doi: $10.6004 /$ jnccn.2021.7065

\footnotetext{
${ }^{1}$ Veterans Health Administration San Diego Health Care System, and ${ }^{2}$ Department of Radiation Medicine and Applied Sciences, ${ }^{3}$ Department of Urology, School of Medicine, University of California, San Diego; ${ }^{4}$ Janssen Pharmaceuticals Research and Development, LCC; and ${ }^{5}$ Division of Hematology-Oncology, Department of Internal Medicine, School of Medicine, University of California, San Diego, La Jolla, California.
}

\section{Background}

Active surveillance (AS) is an appropriate management strategy for patients with localized, NCCN-classified lowrisk prostate cancer. ${ }^{1-6}$ The use of AS in men with intermediate-risk prostate cancer, however, remains unclear. Prior studies of AS in intermediate-risk disease have been limited by small sample sizes, short follow-up, and lack of definitive clinical outcomes, including metastasis and prostate cancer-specific mortality (PCSM) ${ }^{5-12}$ Nevertheless, the use of AS in intermediate-risk prostate cancer has increased in recent years, ${ }^{13}$ and it is now an NCCN-recognized option for men with favorable intermediate-risk prostate cancer. ${ }^{1}$

The US Veterans Health Administration (VHA) is a relatively equal-access healthcare system that provides care to a large, diverse, and predominantly male population and frequently uses AS. ${ }^{14,15}$ In this study, we identified men with low- and intermediate-risk prostate cancer undergoing AS within the VHA and sought to assess clinical outcomes in men treated for intermediate-risk prostate cancer with AS.

\section{Patients and Methods}

\section{Data Source}

We extracted patient data from the VHA's Corporate Data Warehouse (CDW), which contains health records of $>9$ million veterans from approximately 170 VHA medical centers and 1,000 outpatient sites. ${ }^{16}$ This study was reviewed and approved by the VHA San Diego Health Care System. Waivers of consent and authorization were granted by the Institutional Review Board and the Research and Development Committee of the VHA San Diego Health Care System (Protocol Number 150169).

\section{Study Population}

We identified patients with either low- or intermediaterisk prostate cancer diagnosed between January 1, 2001,

See JNCCN.org for supplemental online content. 
and December 31, 2015, in the VHA healthcare system who were initially managed with AS. Prostate cancer risk groups were defined according to NCCN Clinical Practice Guidelines in Oncology (NCCN Guidelines). ${ }^{1}$ Low-risk prostate cancer was defined as clinical tumor stage $\leq 2 \mathrm{~A}$, Gleason score $\leq 6$, and prostate-specific antigen (PSA) level $<10 \mathrm{ng} / \mathrm{mL}$. Intermediate-risk prostate cancer was defined as having $\geq 1$ intermediate-risk feature (clinical tumor stage 2B-C, Gleason score 7, or PSA $10-20 \mathrm{ng} / \mathrm{mL}$ ) and no high-risk or very-high-risk features (clinical tumor stage $\geq 3$, Gleason score $\geq 8$, PSA $>20 \mathrm{ng} / \mathrm{mL}$ ). Intermediate-risk patients were divided into favorable and unfavorable intermediate-risk subgroups. Favorable intermediate risk was defined as having only 1 intermediate-risk feature and a Gleason score $\leq 6$ or $3+4=7$. Unfavorable intermediate risk was defined as having at least 2 intermediate-risk features and/or a Gleason score $4+3=7$. We did not include percent positive biopsy cores in the intermediate-risk subgroup definitions, because it is not consistently ascertained in the VHA CDW. In addition, we did not include patients who were neither African American nor non-Hispanic White to maintain consistency between the low- and intermediate-risk subcohorts, because the low-risk subcohort had been created first and for the purpose of comparing outcomes between African American and non-Hispanic White patients treated with AS. ${ }^{17}$ Race and ethnicity were self-reported and based on fixed categories.

We considered patients to be undergoing AS if they did not receive definitive treatment (prostatectomy or radiation) or androgen deprivation therapy within the first year of prostate cancer diagnosis and underwent at least one additional prostate biopsy after their diagnostic biopsy. ${ }^{17}$ Patients were excluded if they had received pelvic radiation before prostate cancer diagnosis or had missing values for zip code-level median household income and education. Supplemental eFigure 1 (available with this article at JNCCN.org) summarizes our cohort selection process.

\section{Outcomes}

The main endpoints of the study were incidence of definitive treatment with prostatectomy or radiation, metastasis, PCSM, and all-cause mortality. We identified definitive treatment using diagnosis and procedural codes and manual chart review. Inpatient and outpatient data in the VHA CDW and a CDW-linked Centers for Medicare \& Medicaid Services data registry were searched for ICD-9/10 and CPT codes to identify the receipt of radical prostatectomy or curative-intent radiation therapy. We then manually reviewed charts of patients who had $\mathrm{a} \geq 50 \%$ decrease in PSA from baseline at any point after prostate cancer diagnosis to determine whether they had received definitive treatment outside the VHA healthcare system.

We identified metastatic prostate cancer using targeted chart review. We manually reviewed medical charts of patients meeting any of the following criteria: (1) patient records contained ICD-9/10 codes for bone metastases or malignant neoplasms of nonpelvic lymph nodes (supplemental eTable 1), (2) PSA $>20 \mathrm{ng} / \mathrm{mL}$ reported any time after prostate cancer diagnosis, (3) receipt of androgen deprivation therapy, and (4) receipt of a bone scan at least 6 months after prostate cancer diagnosis, identified using ICD-9/10 and CPT codes (supplemental eTable 1). We manually reviewed the charts of 100 patients who did not meet any of those criteria and did not identify any missed cases of metastases.

Finally, PCSM and all-cause mortality outcomes were obtained using National Death Index data linked to the VHA CDW and manual chart review. The National Death Index provided data through December 31, 2015; therefore, we identified patients with a date of death after December 31, 2015, using standard fields within the VHA CDW and performed manual chart review of these patients to determine cause of death.

\section{Statistical Analysis}

Patients were grouped by prostate cancer risk group: low risk, favorable intermediate risk, and unfavorable intermediate risk. Follow-up time was from the date of diagnosis to death or last follow-up. The follow-up cutoff date was March 31, 2020. Pearson chi-square and Wilcoxon rank sum tests were used to identify differences in baseline covariates. We analyzed rates of definitive treatment, metastasis, PCSM, and all-cause mortality using cumulative incidence functions. Gray's test for equality was used to evaluate differences in cumulative incidences.

We assessed the impact of prostate cancer risk group on metastasis and PCSM with multivariable Fine-Gray competing-risks regression. ${ }^{18}$ For metastasis, death of any cause was considered as a competing event. For PCSM, death of non-prostate cancer causes was considered as a competing event. All-cause mortality was assessed with Cox proportional hazards regression.

Regression models incorporated prostate cancer risk group, age at diagnosis, race, Charlson comorbidity index (CCI) score, ${ }^{19}$ statin use, cigarette use, Agent Orange exposure, receipt of definitive treatment, US geographic region, zip code-level median household income and education level (from American Community Survey census data ${ }^{20}$ ), and year of diagnosis. Age was a continuous variable. CCI score was categorized as 0,1 , and $\geq 2$. US geographic region was categorized as West, Midwest, South, and Northeast. Income and education levels (ie, percentage receiving a bachelor's degree) were stratified based on the median values for the cohort $(\$ 48,333$ and 
$14.4 \%$, respectively). Year of diagnosis was grouped into 2001-2005, 2006-2010, and 2011-2015. The association of individual patient biopsy patterns with the risk of metastasis and PCSM was also tested in separate sensitivity analyses in which the following measures were individually incorporated into the multivariable regression models: (1) the total number of biopsies received, (2) the average time between biopsies in years, and (3) the time between the first surveillance biopsy and diagnosis in years.

We used SAS 9.2 (SAS Institute Inc) for all statistical analyses, assuming a 2-sided $\alpha$ of 0.05 . Figures were designed using RStudio version 3.5.1 (RStudio).

\section{Results}

\section{Study Population}

The cohort included 9,733 men with low- or intermediate-risk prostate cancer undergoing AS (Supplemental eFigure 1). A total of 1,007 men (10.3\%) had intermediate-risk disease, including 773 (76.8\%) with favorable intermediate-risk and 234 (23.2\%) with unfavorable intermediate-risk (Table 1). The median follow-up for the entire cohort was 7.6 years (interquartile range, 5.7-9.8 years). A total of 2,309 patients (23.7\%) were followed for at least 10 years, including 2,081 (23.8\%) with low-risk, $178(23.0 \%)$ with favorable intermediate-risk, and 50 (21.4\%) with unfavorable intermediate-risk disease. Follow-up length did not significantly differ between prostate cancer risk groups $(P=.89)$. The median number of PSA tests for the entire cohort was 12 , and there was a nonsignificant difference $(P=.21)$ in the median number of PSA tests between the low-risk $(n=12)$, favorable intermediate-risk $(n=12)$, and unfavorable intermediate-risk $(n=13)$ groups. The median number of biopsies was 2, and there was no difference in the median number of biopsies between the low-risk $(n=2)$, favorable intermediate-risk $(n=2)$, and unfavorable intermediate-risk $(n=2)$ groups.

The median age of the entire cohort was 65.2 years. A total of 2,610 patients (26.8\%) were African American, including 2,280 (26.1\%) low-risk, 252 (32.6\%) favorable intermediate-risk, and 78 (33.3\%) unfavorable intermediate-risk patients $(P<.001$, for all groups). Aside from race, baseline covariates did not substantially differ between prostate cancer risk groups. Most patients were nonsmokers (80.8\%), had a CCI score of $0(77.6 \%)$, did not report exposure to Agent Orange (76.7\%), and had an annual income between $\$ 30,000$ and $\$ 60,000$ (65.5\%), and approximately half (50.4\%) were prescribed statins.

\section{Definitive Treatment}

A total of 4,361 patients (44.8\%) eventually received definitive treatment at a median of 3.1 years from diagnosis.
The median times to definitive treatment of the favorable (2.6 years) and unfavorable (2.6 years) intermediate-risk groups were significantly shorter than that in the low-risk group (3.2 years; $P<.001$, for both). The 10 -year cumulative incidences of definitive treatment were $44.9 \%, 81.6 \%$, and $78.5 \%$ for the low-risk, favorable intermediate-risk, and unfavorable intermediate-risk groups, respectively (Table 2).

Among those with intermediate-risk disease, 509 (50.5\%; 389 favorable, 120 unfavorable) received radiation, 277 (27.5\%; 218 favorable, 59 unfavorable) received prostatectomy, and 221 (21.9\%; 166 favorable, 55 unfavorable) received no definitive treatment. The proportions of types of definitive treatment received did not significantly differ between the favorable and unfavorable intermediate-risk groups $(P=.62)$.

\section{Metastasis}

A total of 199 men experienced metastasis, including 109 with low-risk, 55 with favorable intermediate-risk, and 35 with unfavorable intermediate-risk. The 10-year cumulative incidence of metastasis was $1.5 \%$ for low-risk patients, $9.6 \%$ for favorable intermediate-risk patients, and $19.2 \%$ for unfavorable intermediate-risk patients (Table 2, Figure 1A) $(P<.001$ for all groups and low vs favorable intermediate-risk groups). In multivariable Fine-Gray regression, those with favorable (subdistribution hazard ratio [SHR], 6.49; 95\% CI, 4.39-9.59; $P<.001$ ) and unfavorable (SHR, 14.45; 95\% CI, 9.27-22.52; $P<.001$ ) intermediate-risk disease had a significantly increased risk for developing metastases compared with those with low-risk disease (Table 3). In the sensitivity analyses incorporating biopsy patterns, an increase in the total number of biopsies was not significantly associated with the risk for developing metastases (SHR, 0.86; 95\% CI, $0.72-1.02 ; P=.08)$. An increase in the average time between biopsies (SHR, 1.16; 95\% CI, 1.09-1.24; $P<.001$ ) and the time to first surveillance biopsy (SHR, 1.13; 95\% CI, 1.06-1.20; $P<.001$ ) were both significantly associated with an increased risk for developing metastases.

\section{Mortality}

During the study period, 130 patients died of prostate cancer, including 87 with low-risk, 24 with favorable intermediate-risk, and 19 with unfavorable intermediaterisk. The 10-year cumulative incidence of PCSM was $1.1 \%$ for low-risk, $3.7 \%$ for favorable intermediate-risk, and $11.8 \%$ for unfavorable intermediate-risk patients (Table 2, Figure 1B) $(P<.001$ for all groups and low vs favorable intermediate-risk groups). In multivariable Fine-Gray regression, those with favorable (SHR, 2.94; 95\% CI, 1.83-4.73; $P<.001$ ) and unfavorable (SHR, 7.90; 95\% CI, 4.63-13.50; $P<.001)$ intermediate-risk disease had a significantly increased risk for experiencing death 


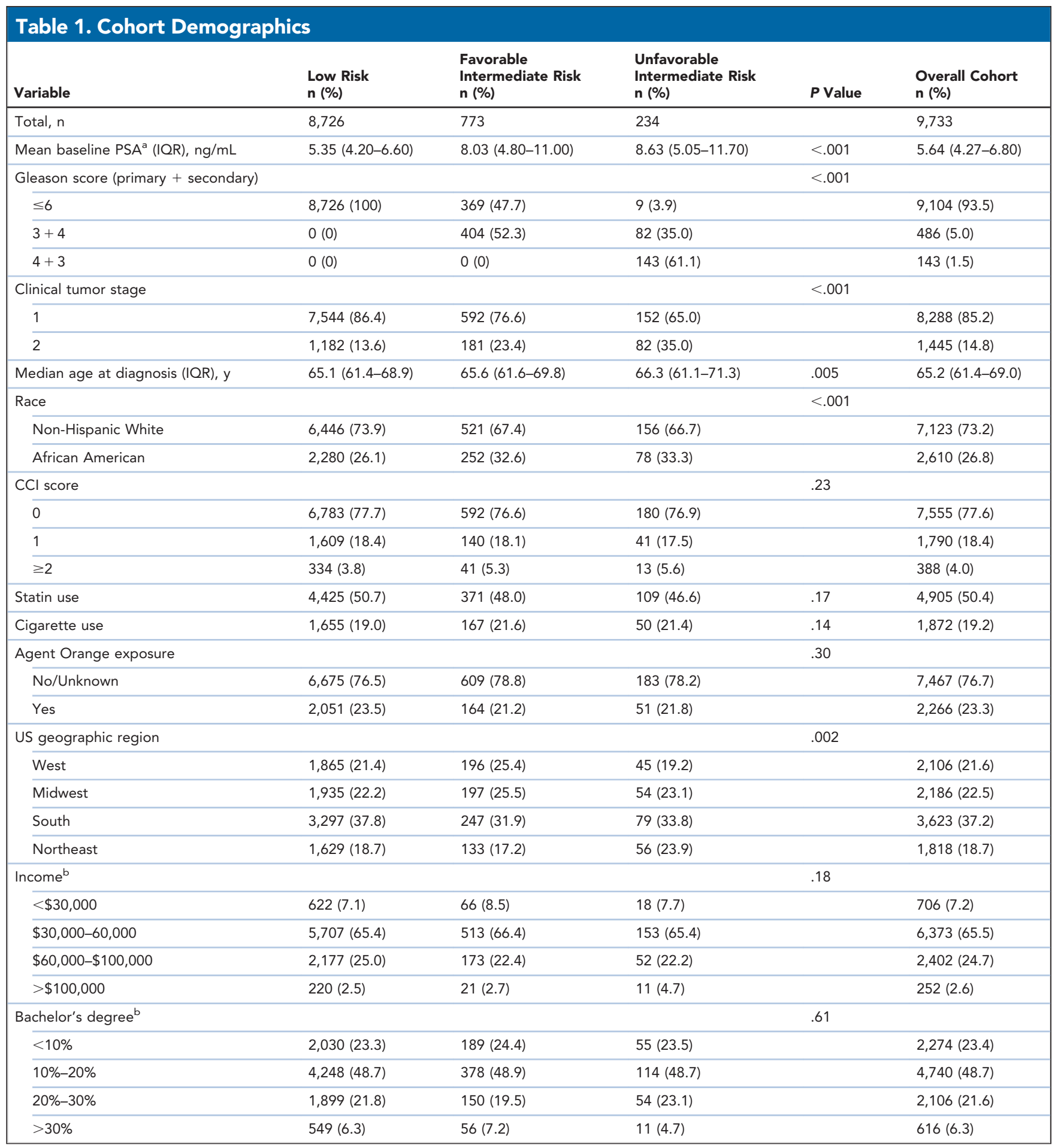

of prostate cancer compared with those with low-risk disease (Table 3). In the sensitivity analyses incorporating biopsy patterns, an increase in the total number of biopsies was significantly associated with a decreased risk for PCSM (SHR, 0.76; 95\% CI, 0.60-0.98; $P=.03$ ).
An increase in the average time between biopsies (SHR, 1.05; 95\% CI, $0.98-1.14 ; P=.19$ ) and time to first surveillance biopsy (SHR, 1.06; 95\% CI, $0.99-1.14 ; P=.09$ ) were both not significantly associated with risk for PCSM. 


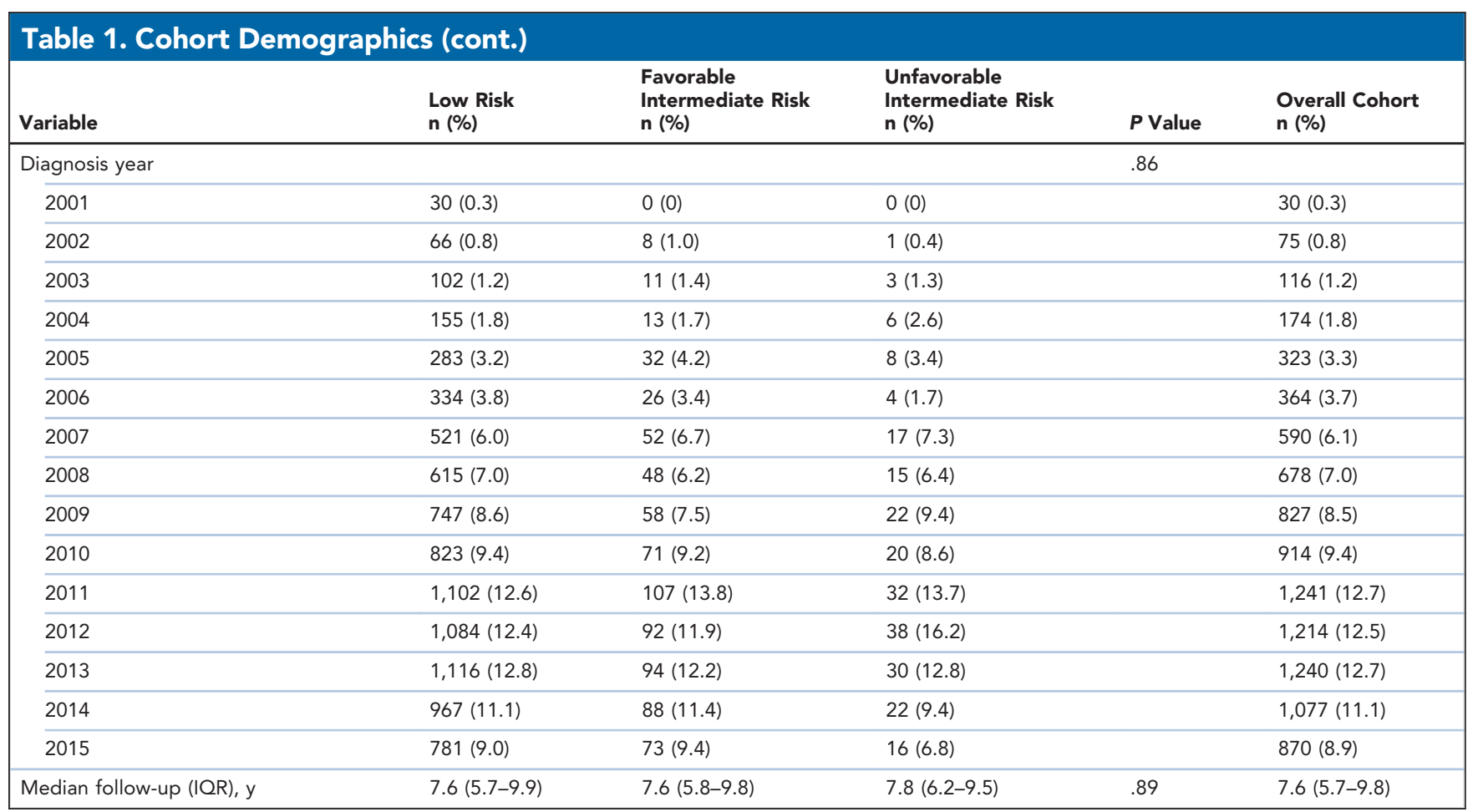

Abbreviations: $\mathrm{CCl}$, Charlson comorbidity index; IQR, interquartile range; PSA, prostate-specific antigen

aPSA value closest to and before prostate cancer diagnosis.

${ }^{b}$ Based on zip code-level values. Income was measured as the median zip code value. Education was defined as the percentage of those living within a zip code possessing a bachelor's degree.

A total of 1,983 patients died of any cause, including 1,739 with low-risk, 171 with favorable intermediate-risk, and 73 with unfavorable intermediate-risk disease. The 10-year cumulative incidences of allcause mortality were $23.2 \%, 26.2 \%$, and $40.6 \%$ for the low-risk, favorable intermediate-risk, and unfavorable intermediate-risk groups, respectively (Table 2) $(P<.001$ for all groups; $P=.13$ between low- and favorable intermediate-risk groups). In multivariable Cox proportional hazards regression, those with favorable intermediate-risk disease (HR, 1.13; 95\% CI, 0.96-1.32; $P=.13$ ) did not have a significantly increased risk for all-cause mortality, although those with unfavorable intermediate-risk disease (HR, 1.61; 95\% CI, 1.28-2.03; $P<.001)$ did have a significantly increased risk compared with low-risk patients.

\section{Discussion}

In this large, national cohort of men with prostate cancer undergoing AS, we found that both favorable and unfavorable intermediate-risk patients were at significantly increased risk for experiencing metastases and death due to prostate cancer compared with low-risk patients. These data suggest that AS may be an appropriate option for carefully selected African American or non-Hispanic White men with favorable intermediate-risk prostate

\section{Table 2. Ten-Year Cumulative Incidence of Selected Outcomes}

\begin{tabular}{|c|c|c|c|c|c|}
\hline \multirow[b]{2}{*}{ Outcome } & \multicolumn{3}{|c|}{ 10-Year Cumulative Incidence $(95 \% \mathrm{Cl})$} & \multicolumn{2}{|c|}{ Gray's Test $P$ Value } \\
\hline & Low Risk & $\begin{array}{l}\text { Favorable } \\
\text { Intermediate } \\
\text { Risk }\end{array}$ & $\begin{array}{l}\text { Unfavorable } \\
\text { Intermediate } \\
\text { Risk }\end{array}$ & $\begin{array}{l}\text { All Risk } \\
\text { Groups }\end{array}$ & $\begin{array}{l}\text { Low vs Favorable } \\
\text { Intermediate Risk }\end{array}$ \\
\hline Metastasis & $1.5 \%(1.2-1.9)$ & $9.6 \%(7.1-12.5)$ & $19.2 \%(13.4-25.9)$ & $<.001$ & $<.001$ \\
\hline Prostate cancer-specific mortality & $1.1 \%(0.8-1.4)$ & $3.7 \%(2.3-5.7)$ & $11.8 \%(6.8-18.4)$ & $<.001$ & $<.001$ \\
\hline
\end{tabular}

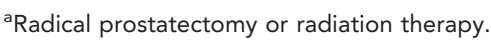


A

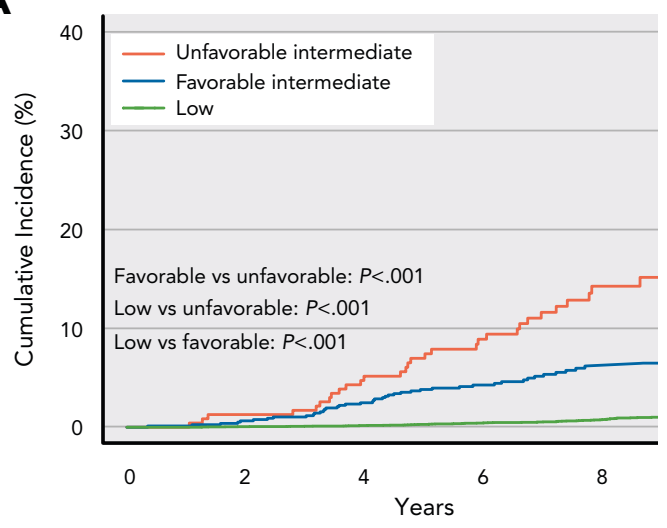

B

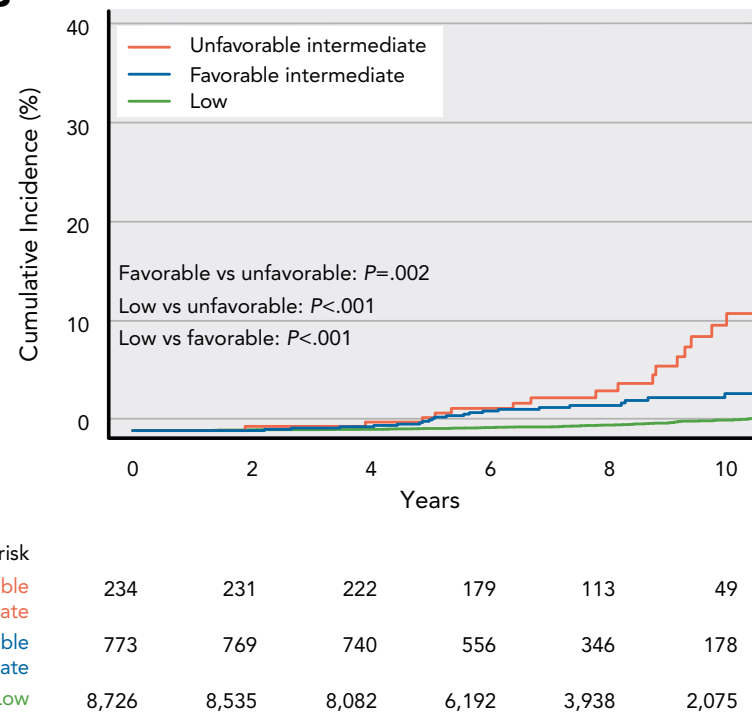

Figure 1. Cumulative incidences of (A) metastasis and (B) prostate cancer-specific mortality, with Gray's test for equality $P$ values between prostate cancer risk groups.

cancer, but that the risks for favorable intermediate-risk patients are significantly higher than suggested by the literature on predominantly low-risk patients.

Current NCCN Guidelines for Prostate Cancer (Version 2.2021) consider AS an option for men with favorable intermediate-risk disease despite a relative lack of data in this risk group. ${ }^{1}$ The recently published ProtecT randomized trial did include patients with intermediaterisk disease, but metastasis and PCSM rates were not reported by risk group, and the favorable outcomes may reflect the predominance of low-risk patients. ${ }^{5}$ Large prospective cohorts of patients undergoing AS have also typically included primarily low-risk patients. Subgroup analyses of intermediate-risk patients from these trials have reported 10-year metastasis-free survival rates of $91 \%$ (all intermediate risk) to $92 \%$ (favorable intermediate risk) ${ }^{7}$ and $96.4 \%,{ }^{8}$ along with 10 -year prostate cancer-specific survival rates of $96.1 \%,{ }^{8} 97 \%,{ }^{7}$ and $98 \%{ }^{9}$ Sample sizes in these reports were generally small, consisting of approximately 100 to 200 patients, and likely skewed toward favorable intermediate-risk disease. ${ }^{7-9}$ With 10 -year cumulative incidences of metastasis and PCSM of $9.6 \%$ and 3.7\%, respectively, for men with favorable intermediate-risk prostate cancer, our results are broadly in line with the prior studies. The slight increase may be a result of less stringent entry criteria or less strict surveillance as practiced in a community setting rather than an active protocol. Together these data show consistently higher risks than those seen with AS for low-risk disease.

Given the heterogeneity of the disease factors used to determine risk group, a subset of favorable intermediate-risk patients may exist for whom AS could be considered safe. Those harboring any Gleason pattern 4 disease have been shown to have worse outcomes ${ }^{12,21-23}$ and increased rates of adverse pathologic findings ${ }^{21,24-26}$ compared with those with only Gleason pattern 3 disease, even though patients with either Gleason $3+4=7$ or $3+3=6$ can be categorized as favorable intermediate-risk patients. Similarly, other factors may contribute to the risk for worse oncologic outcomes. Although not measured in this study, molecular testing and genomic risk stratification represent potential methods for improving the ability to determine which patients are more suitable for AS. ${ }^{27,28}$ In addition, improved surveillance protocols may improve outcomes. Our sensitivity analyses of biopsy patterns found that the number of or the time between biopsies was associated with the risks for PCSM and metastasis, respectively. However, validation of these findings requires additional prospective evaluation, and methods for identifying the "ideal" favorable intermediate-risk candidate for AS must be carefully tested in future studies.

Patients with unfavorable intermediate-risk disease were at substantially higher risk for metastases and PCSM. Existing data for this group are particularly limited, with the Sunnybrook cohort ${ }^{7}$ reporting a 15-year metastasis-free survival rate of $63 \%$ for 20 men with Gleason $4+3=7$ disease. Notably, the unfavorable intermediate-risk group was relatively young and healthy (by CCI score) in the present study, which may be unexpected in light of current NCCN Guidelines that do not recommend AS for unfavorable intermediate-risk prostate 


\section{Table 3. Multivariable Fine-Gray Competing-Risks Regression Analysis of Metastasis and PCSM}

\begin{tabular}{|c|c|c|c|c|}
\hline \multirow[b]{2}{*}{ Characteristic } & \multicolumn{2}{|c|}{ Metastasis } & \multicolumn{2}{|c|}{ PCSM } \\
\hline & SHR $(95 \% \mathrm{Cl})$ & $P$ Value & SHR (95\% Cl) & $P$ Value \\
\hline \multicolumn{5}{|l|}{ NCCN risk group } \\
\hline Low & Ref & & Ref & \\
\hline Favorable intermediate & $6.49(4.39-9.59)$ & $<.001$ & $2.94(1.83-4.73)$ & $<.001$ \\
\hline Unfavorable intermediate & $14.45(9.27-22.52)$ & $<.001$ & $7.90(4.63-13.50)$ & $<.001$ \\
\hline Age at diagnosis ${ }^{a}$ & $1.00(0.98-1.02)$ & .97 & $1.05(1.02-1.07)$ & .001 \\
\hline \multicolumn{5}{|l|}{ Race } \\
\hline Non-Hispanic White & Ref & & Ref & \\
\hline African American & $1.03(0.74-1.44)$ & .87 & $1.29(0.86-1.94)$ & .21 \\
\hline \multicolumn{5}{|l|}{$\mathrm{CCl}$ score } \\
\hline 0 & Ref & & Ref & \\
\hline 1 & $0.99(0.68-1.44)$ & .96 & $1.16(0.75-1.80)$ & .50 \\
\hline$\geq 2$ & $1.82(1.06-3.15)$ & .03 & $1.49(0.71-3.13)$ & .29 \\
\hline \multicolumn{5}{|l|}{ Diagnosis year } \\
\hline 2001-2005 & Ref & & Ref & \\
\hline $2006-2010$ & $0.89(0.60-1.32)$ & .56 & $0.51(0.34-0.77)$ & .001 \\
\hline $2011-2015$ & $0.66(0.43-1.02)$ & .06 & $0.27(0.16-0.47)$ & $<.001$ \\
\hline \multicolumn{5}{|l|}{ Statin use } \\
\hline No & Ref & & Ref & \\
\hline Yes & $1.14(0.86-1.52)$ & .37 & $1.02(0.71-1.44)$ & .93 \\
\hline \multicolumn{5}{|l|}{ Cigarette use } \\
\hline No & Ref & & Ref & \\
\hline Yes & $1.25(0.88-1.76)$ & .22 & $1.19(0.74-1.93)$ & .47 \\
\hline \multicolumn{5}{|l|}{ Agent Orange exposure } \\
\hline No/Unknown & Ref & & Ref & \\
\hline Yes & $1.20(0.85-1.69)$ & .30 & $1.24(0.78-1.99)$ & .36 \\
\hline \multicolumn{5}{|l|}{ Definitive treatment } \\
\hline No & Ref & & Ref & \\
\hline Yes & $0.74(0.52-1.04)$ & .09 & $1.03(0.71-1.49)$ & .87 \\
\hline \multicolumn{5}{|l|}{ US geographic region } \\
\hline West & Ref & & Ref & \\
\hline Midwest & $1.00(0.68-1.49)$ & .98 & $0.99(0.61-1.61)$ & .96 \\
\hline South & $0.77(0.52-1.13)$ & .18 & $0.89(0.57-1.40)$ & .62 \\
\hline Northeast & $0.79(0.51-1.23)$ & .30 & $0.53(0.29-0.96)$ & .04 \\
\hline \multicolumn{5}{|l|}{ Income ${ }^{b}$} \\
\hline$<\$ 48,333^{c}$ & Ref & & Ref & \\
\hline$\geq \$ 48,333$ & $1.08(0.76-1.53)$ & .68 & $1.03(0.67-1.59)$ & .88 \\
\hline \multicolumn{5}{|l|}{ Bachelor's degree $^{b}$} \\
\hline$<14.4 \%^{\mathrm{c}}$ & Ref & & Ref & \\
\hline$\geq 14.4 \%$ & $0.94(0.67-1.32)$ & .71 & $0.99(0.66-1.50)$ & .98 \\
\hline
\end{tabular}

Abbreviations: $\mathrm{CCl}$, Charlson comorbidity index; PCSM, prostate cancer-specific mortality; SHR, subdistribution hazard ratio.

${ }^{a}$ Represents SHR for 1-year increase in age at diagnosis.

${ }^{\mathrm{b}}$ Based on zip code-level values. Income was measured as the median zip code value. Education was defined as the percentage within a zip code possessing a bachelor's degree.

'Total study cohort median. 
cancer and do recommend only observation for those with unfavorable intermediate-risk disease with a life expectancy $<10$ years. ${ }^{1}$ Although we are unable to determine the exact reasons for which AS was selected for these patients, the division of the intermediate-risk group into the favorable and unfavorable subgroups and subsequent treatment recommendations were adopted relatively recently ${ }^{29,30}$ and after most of the unfavorable intermediate-risk patients included in this study were diagnosed. Overall, the present study supports the existing literature suggesting that unfavorable intermediate-risk disease has a more aggressive disease course, ${ }^{31}$ further supporting NCCN Guidelines recommending against AS for unfavorable intermediate-risk patients. ${ }^{1}$

Our study had potential limitations worth noting. As a retrospective study, it had no predefined AS protocol. We required that all patients had at least one additional prostate biopsy in an attempt to differentiate and exclude watchful waiting patients. In addition, the high rates of definitive treatment in the intermediate-risk group suggest that these patients were being actively surveilled with the intention to treat if their cancer progressed. However, we were unable to determine the indications for or reason(s) why a patient ultimately underwent treatment. Subsequent clinical decisions were ultimately made at the discretion of the physicians and their patients, although we did not detect any significant differences in the rates of follow-up PSA tests and biopsies between risk groups or races or in the rates of definitive treatment between the intermediate-risk subgroups. In addition, there was no prespecified metastasis screening protocol. It is possible that some patients with metastases were missed because they did not undergo the clinical evaluation that would have identified them for manual chart review. However, we do not think that this would have been different across comparison groups, and our rates of metastasis and PCSM are generally in line with previous studies. Furthermore, we did not measure the use of multiparametric MRI of the prostate, which is increasingly used in risk stratification for and monitoring during $\mathrm{AS}^{32-34}$ and not considering its use in the present study might have contributed to our observed rates of clinical outcomes. Finally, omitting the percentage of positive biopsy cores from our intermediate-risk subgroup definitions is a limitation. This histopathologic factor, among others not measured in this study, such as percentage of Gleason pattern 4 involvement or other growth patterns, has been shown to be associated with the risk of certain oncologic outcomes, ${ }^{12,21-26,35}$ and thus could have influenced our results. Moreover, this omission could have resulted in misclassification of unfavorable intermediate-risk patients as favorable intermediate-risk.

\section{Conclusions}

Overall, this study provides important estimates of the risks of AS for men with intermediate-risk prostate cancer to better inform shared decision-making between patients and healthcare providers. Improved patient selection and surveillance protocols may be needed to reduce the risks of AS for men with favorable intermediate-risk prostate cancer. Prospective studies are needed to further define the safest clinical practices for AS and to validate these findings.

Submitted February 8, 2021; final revision received May 27, 2021; accepted for publication May 27, 2021.

Previous presentation: A version of this project was presented as a quick pitch oral abstract at the ASTRO 2020 Annual Meeting; October 24-28, 2020; Miami, Florida [Int J Radiat Oncol Biol Phys 2020;108(3 Suppl): S121-122].

Author contributions: Conceptualization: Parsons, Rose. Data curation Courtney, Deka, Cherry, Salans, Nelson, Kumar, Luterstein, Yip, Nalawade. Formal analysis: Courtney, Deka, Nalawade, Rose. Funding acquisition: Courtney, Deka, Cherry, Salans, Yip, Rose. Investigation: Courtney, Deka, Kotha, Cherry, Salans, Nelson, Kumar, Luterstein, Yip. Methodology: Courtney, Deka, Kotha, Nalawade, Kader, Stewart, Rose. Project administration: Courtney, Deka, Rose. Resources: Rose. Software: Courtney, Deka, Nalawade. Supervision: Parsons, Kader, Stewart, Rose. Validation: Deka, Nelson, Parsons, Kader, Stewart. Visualization: Courtney, Deka, Nelson, Rose. Writing - original draft: Courtney, Rose. Writing - review and editing: Courtney, Kotha, Cherry, Salans, Nelson, Kumar, Luterstein, Yip, Nalawade, Parsons, Kader, Stewart, Rose.

Disclosures: Dr. Kumar has disclosed receiving previous compensation from Sympto Health. Dr. Parsons has disclosed serving as an advisory board member for Janssen Pharmaceutica Products, LP. Dr. Stewart has disclosed serving as an advisory board member for Seagen/Astellas and receiving research funding from GRAIL. The remaining authors have disclosed that they have not received any financial consideration from any person or organization to support the preparation, analysis, results, or discussion of this article.

Funding: Research reported in this publication was supported by the NIH under grant number TL1-TR001443 (P.T. Courtney, D.R. Cherry, M.A. Salans, A.T. Yip) and by the U.S. Department of Defense under grant number W81XWH-17-PCRP-PRA (R. Deka, B.S. Rose).

Disclaimer: The content is solely the responsibility of the authors and does not necessarily represent the official views of the $\mathrm{NIH}$.

Correspondence: Brent S. Rose, MD, Department of Radiation Medicine and Applied Sciences, Altman Clinical and Translational Research Institute, University of California, San Diego, 9452 Medical Center Drive, La Jolla, CA 92037. Email: bsrose@health.ucsd.edu

\section{References}

1. Schaeffer E, Srinivas S, Antonarakis ES, et al. NCCN Clinical Practice Guidelines in Oncology: Prostate Cancer. Version 2.2021. Accessed May 27, 2021. To view the most recent version, visit NCCN.org
2. Bul $M$, Zhu $X$, Valdagni $R$, et al. Active surveillance for low-risk prostate cancer worldwide: the PRIAS study. Eur Urol 2013;63: 597-603. 
3. Tosoian JJ, Carter HB, Lepor A, et al. Active surveillance for prostate cancer: current evidence and contemporary state of practice. Nat Rev Urol 2016;13:205-215

4. Tosoian JJ, Mamawala M, Epstein JI, et al. Intermediate and longer-term outcomes from a prospective active-surveillance program for favorablerisk prostate cancer. J Clin Oncol 2015;33:3379-3385.

5. Hamdy FC, Donovan JL, Lane JA, et al. 10-year outcomes after monitoring, surgery, or radiotherapy for localized prostate cancer. N Engl J Med 2016;375:1415-1424

6. Klotz L, Vesprini D, Sethukavalan $P$, et al. Long-term follow-up of a large active surveillance cohort of patients with prostate cancer. J Clin Oncol 2015;33:272-277

7. Musunuru HB, Yamamoto T, Klotz L, et al. Active surveillance for intermediate risk prostate cancer: survival outcomes in the Sunnybrook experience. J Urol 2016;196:1651-1658.

8. Bul M, van den Bergh RC, Zhu X, Loeb S. Outcomes of initially expectantly managed patients with low or intermediate risk screen-detected localized prostate cancer. BJU Int 2012;110:1672-1677.

9. Godtman RA, Holmberg E, Khatami A, et al. Long-term results of active surveillance in the Göteborg randomized, population-based prostate cancer screening trial. Eur Urol 2016;70:760-766.

10. Cooperberg MR, Cowan JE, Hilton JF, et al. Outcomes of active surveillance for men with intermediate-risk prostate cancer. J Clin Oncol 2011; 29:228-234

11. Selvadurai ED, Singhera M, Thomas $K$, et al. Medium-term outcomes of active surveillance for localised prostate cancer. Eur Urol 2013;64:981-987.

12. Yamamoto $T$, Musunuru HB, Vesprini $D$, et al. Metastatic prostate cancer in men initially treated with active surveillance. J Urol 2016;195:1409-1414.

13. Butler SS, Mahal BA, Lamba N, et al. Use and early mortality outcomes of active surveillance in patients with intermediate-risk prostate cancer. Cancer 2019;125:3164-3171.

14. Filson CP, Shelton JB, Tan HJ, et al. Expectant management of veterans with early-stage prostate cancer. Cancer 2016;122:626-633.

15. Loeb S, Byrne N, Makarov DV, et al. Use of conservative management for low-risk prostate cancer in the Veterans Affairs integrated health care system from 2005-2015. JAMA 2018;319:2231-2233.

16 U.S. Department of Veteran Affairs. Veterans Health Administration. About VHA. Accessed May 27, 2021. Available at: https://www.va.gov/ health/aboutvha.asp

17. Deka R, Courtney PT, Parsons JK, et al. Association between African American race and clinical outcomes in men treated for low-risk prostate cancer with active surveillance. JAMA 2020;324:1747-1754.

18. Fine JP, Gray RJ. A proportional hazards model for the subdistribution of a competing risk. J Am Stat Assoc 1999;94:496-509.

19. Charlson M, Szatrowski TP, Peterson J, Gold J. Validation of a combined comorbidity index. J Clin Epidemiol 1994;47:1245-1251.
20. Coogan PF, Castro-Webb N, Yu J, et al. Neighborhood and individual socioeconomic status and asthma incidence in African American women. Ethn Dis 2016;26:113-122

21. Gearman DJ, Morlacco A, Cheville JC, et al. Comparison of pathological and oncologic outcomes of favorable risk Gleason score $3+4$ and low risk Gleason score 6 prostate cancer: considerations for active surveillance. J Urol 2018;199:1188-1195.

22. Masic S, Cowan JE, Washington SL, et al. Effects of initial Gleason grade on outcomes during active surveillance for prostate cancer. Eur Urol Oncol 2018;1:386-394.

23. Perlis N, Sayyid R, Evans A, et al. Limitations in predicting organ confined prostate cancer in patients with Gleason pattern 4 on biopsy: implications for active surveillance. J Urol 2017;197:75-83.

24. Aghazadeh MA, Frankel J, Belanger M, et al. National Comprehensive Cancer Network favorable intermediate risk prostate cancer-is active surveillance appropriate? J Urol 2018;199:1196-1201.

25. Patel HD, Tosoian JJ, Carter HB, Epstein Jl. Adverse pathologic findings for men electing immediate radical prostatectomy: defining a favorable intermediate-risk group. JAMA Oncol 2018;4:89-92.

26. Loeb S, Folkvaljon $Y$, Bratt $O$, et al. Defining intermediate risk prostate cancer suitable for active surveillance. J Urol 2019;201:292-299.

27. Chung JS, Morgan TM, Hong SK. Clinical implications of genomic evaluations for prostate cancer risk stratification, screening, and treatment: a narrative review. Prostate Int 2020;8:99-106.

28. Eggener SE, Rumble RB, Armstrong AJ, et al. Molecular biomarkers in localized prostate cancer: ASCO guideline. J Clin Oncol 2020;38:1474-1494.

29. Zumsteg ZS, Spratt DE, Pei I, et al. A new risk classification system for therapeutic decision making with intermediate-risk prostate cancer patients undergoing dose-escalated external-beam radiation therapy. Eur Urol 2013;64:895-902.

30. Mohler JL, Armstrong AJ, Bahnson RR, et al. NCCN Guidelines Insights: Prostate Cancer, Version 1.2016. J Natl Compr Canc Netw 2016;14:19-30.

31. Preisser F, Cooperberg MR, Crook J, et al. Intermediate-risk prostate cancer: stratification and management. Eur Urol Oncol 2020;3:270-280.

32. Chiam K, Carle C, Hughes $S$, et al. Use of multiparametric magnetic resonance imaging (mpMRI) in active surveillance for low-risk prostate cancer: a scoping review on the benefits and harm of mpMRI in different biopsy scenarios. Prostate Cancer Prostatic Dis 2021;24:662-673.

33. Fam MM, Yabes JG, Macleod LC, et al. Increasing utilization of multiparametric magnetic resonance imaging in prostate cancer active surveillance. Urology 2019;130:99-105.

34. Stavrinides V, Giganti F, Emberton M, Moore CM. MRI in active surveillance: a critical review. Prostate Cancer Prostatic Dis 2019:22:5-15.

35. Balakrishnan AS, Cowan JE, Cooperberg MR, et al. Evaluating the safety of active surveillance: outcomes of deferred radical prostatectomy after an initial period of surveillance. J Urol 2019;202:506-510. 
Supplemental online content for:

\section{Metastasis and Mortality in Men With Low- and Intermediate-Risk Prostate Cancer on Active Surveillance}

P. Travis Courtney, MD, MAS; Rishi Deka, PhD; Nikhil V. Kotha, BS; Daniel R. Cherry, MD, MAS; Mia A. Salans, MD, MAS; Tyler J. Nelson, BS; Abhishek Kumar, MD, MAS; Elaine Luterstein, BS; Anthony T. Yip, MD, MAS; Vinit Nalawade, MS; J. Kellogg Parsons, MD, MHS; A. Karim Kader, MD, PhD; Tyler F. Stewart, MD; and Brent S. Rose, MD

J Natl Compr Canc Netw 2022;20(2):151-159

eFigure 1: Active Surveillance Cohort Selection Process

eTable 1: Medical Billing Codes Used to Identify Patients for Manual Chart Review 


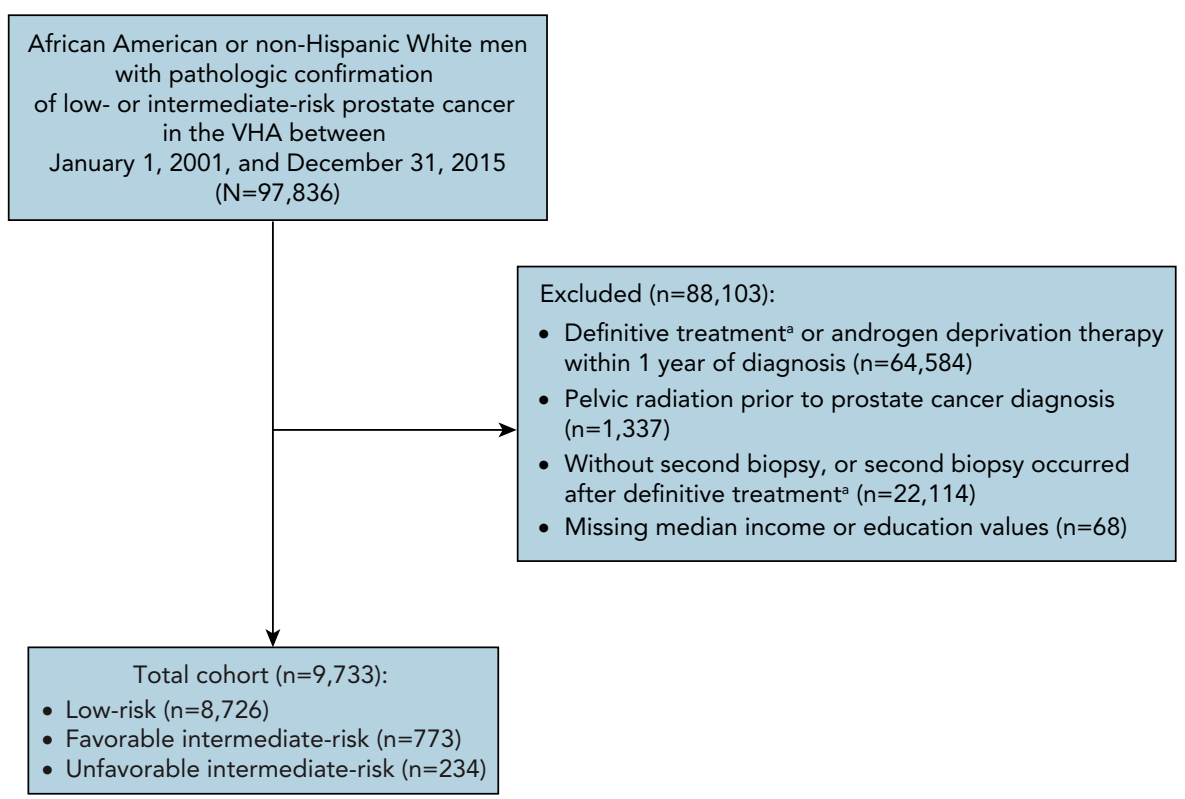

eFigure 1. Active surveillance cohort selection process.

Abbreviation: VHA, Veterans Health Administration.

${ }^{a}$ Radical prostatectomy or radiation therapy. 
eTable 1. Medical Billing Codes Used to Identify Patients for Manual Chart Review for Development of Metastatic Prostate Cancer

\begin{tabular}{|c|c|}
\hline Metastasis Chart Review Criteria & Identification Codes \\
\hline \multirow[t]{2}{*}{ Bone metastases } & ICD-9 diagnosis code: 198.5 \\
\hline & ICD-10 diagnosis codes: C79.51, C79.52 \\
\hline \multirow[t]{2}{*}{ Malignant neoplasm of nonpelvic lymph nodes } & ICD-9 diagnosis codes: $196.0,196.1,196.2,196.3,196.5,196.8,196.9$ \\
\hline & $\begin{array}{l}\text { ICD-10 diagnosis codes: C77.0, C77.1, C77.2, C77.3, C77.4, C77.8, } \\
\text { C77.9 }\end{array}$ \\
\hline \multirow[t]{3}{*}{ Bone scan } & ICD-9 procedure codes: 92.14 \\
\hline & $\begin{array}{l}\text { ICD-10 procedure codes: CP111ZZ, CP1(4-9)1ZZ, CP11YZZ, } \\
\text { CP1(4-9)YZZ, CP1B1ZZ, CP1C1ZZ, CP1D1ZZ, CP1F1ZZ, CP1BYZZ, } \\
\text { CP1CYZZ, CP1DYZZ, CP1FYZZ, CP1YYZZ, CP1Z1ZZ, CP1ZYZZ, } \\
\text { CP2(1-4)1ZZ, CP2(1-4)YZZ, CP2(6-9)1ZZ, CP2(6-9)YZZ, CP2B1ZZ, } \\
\text { CP2BYZZ, CP2C1ZZ, CP2CYZZ, CP2D1ZZ, CP2DYZZ, CP2F1ZZ, } \\
\text { CP2FYZZ, CP2G1ZZ, CP2GYZZ, CP2H1ZZ, CP2HYZZ, CP2J1ZZ, } \\
\text { CP2JYZZ, CP2YYZZ }\end{array}$ \\
\hline & CPT code: 78306 \\
\hline
\end{tabular}

